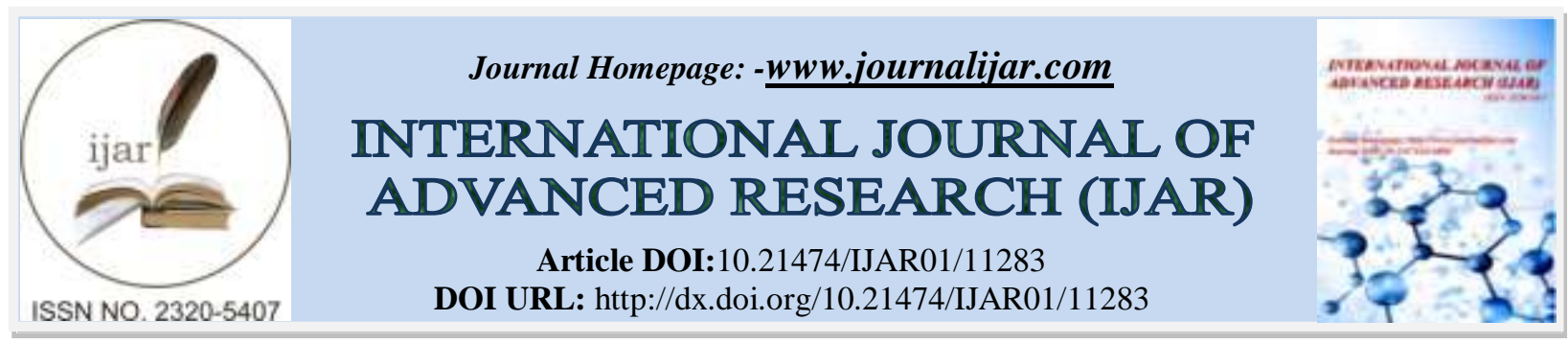

RESEARCH ARTICLE

\title{
AETIOLOGIES AND MANAGEMENT OF URETHRITIS IN MEN: A SYSTEMATIC REVIEW AND META-ANALYSIS
}

Faisal Alsenani, Baraa Kalim, Shahad Aljohani, Faisal Alshehri and Hossam Alnowahi

\section{Manuscript Info}

Manuscript History

Received: 05 May 2020

Final Accepted: 10 June 2020

Published: July 2020

\begin{abstract}
Chlamydia trachomatis and Neisseria gonorrhoea are amongst the most common causes for urethritis and epididymitis in men. Management consists of taking a complete sexual history, examinations of the genital area, assessment of laboratory proof of infection, antimicrobial treatment guided towards the significant aetiologies, and therapy of sexual partners. Therapy of N. gonorrhoea calls for making use of a single-dose program targeting this microorganism, plus a 7- to 10-day tetracycline regimen against $C$. trachomatis and non-gonococcal urethritis. Microbiologic failure is seldom seen in patients taking the proper treatment regimens. However, recurrent urethritis is commonly seen nevertheless. Patients with consistent or recurring symptoms require a cautious re-evaluation, and re-treatment if cultures of polymorphonuclear leukocytes were found in urethral secretions. Although some patients will stay symptomatic and have an increased number of polymorphonuclear leukocytes in urethral secretions, additional treatment is typically not indicated. One of the most vital causes of prostatitis and epididymitis in older males or males with structural anomalies of the urethra are classic urinary system microorganisms as opposed to sexually transferred pathogens. Treatment of these infections consists of getting laboratory evidence of infection and medications against specific pathogens. Men with signs of "prostatitis" need to be assessed making use of both urine and prostatic secretions to look for infection and inflammation. Most of the males with these signs do not have an infection that can be laboratory documented. These patients react poorly to the medication regimen. Males with recorded persistent microbial prostatitis need lengthy regimens of antimicrobials to have an effective treatment. In many cases, nevertheless, the illness is resistant, and persistent treatment with antimicrobials might be needed for the best outcome. Our aim in this article, we will review the causes of urethritis in men and the management plan to treat this common disease.
\end{abstract}

Copy Right, IJAR, 2020,. All rights reserved.

\section{Introduction:-}

Gonococcal and nongonococcal urethritis are one of the most common venereal diseases in males. Failure to manage these infections is an outcome of incorrect therapy in patients and their sexual partners. Sexually transmitted diseases (STDs) are prevalent in the United States and considered an epidemic. Current public exposure has 
concentrated on herpes genitals and the deadly acquired immunodeficiency syndrome (AIDS). Awareness in these incurable conditions has eclipsed the ongoing relevance of more prevalent STDs. Urethritis continues to be one of the most typical conditions found in males with STD. Every year, around 2 million cases of gonorrhoea and 2.5 million cases of non-gonococcal urethritis (NGU) are reported in the U.S. Significant advances have been made in the definition of the aetiologies, epidemiology, and management of NGU. Regardless, several physicians still associate all urethral signs with gonorrhoea and the indication for intramuscular penicillin. Brandt emphasized the prompt requirement for trained front-line physicians to "battle the STD epidemic of the 1980s and 1990s". Nowadays, less than 6 training hours are committed to teaching STDs in a typical four-year clinical college educational program. In this paper, we plan to list the causes and the appropriate management of the male individual with urethral signs and symptoms [1].

C. trachomatis and N. gonorrhoea are the main bacteria found in males with urethritis. Chlamydia is one of the most typically reported STI in the United States, with incidence 3 times as numerous as gonorrhoea. A countrywide depictive sample found a 4 percent incidence of chlamydia in young people, with an approximated 2.8 million cases in the United States annually who are primarily asymptomatic. Although chlamydia can create impediments in men, such as joint inflammation with reactive urethritis (previously referred to as Reiter syndrome), this occurs in only about 1 percent of patients. One research identified a mild increase in prostatitis and a fourfold risk of epididymitis occurrence after a chlamydial infection, however no increase in consequent male infertility reported. Among the major objectives of therapy is to identify the aetiology correctly and to treat sexual partners to stop transmission and its sequelae, such as pelvic inflammatory illness [2].

\section{Aetiology:}

The Centers for Disease Control and Prevention (CDC) approximates that at least 700,000 individuals in the United States contract gonorrhoea yearly. In the United States, the incidence of gonorrhoea is decreasing, although there was a small rise in cases reported in 2006 as well as 2007 prior to decreasing once more in 2008. There is a significant racial difference in the United States, with gonorrhoea rates 40 times greater in 15 to 19 -year-old black men than in white men of the same age. The research study populace showed that genital gonococcal infection is asymptomatic in approximately 10 percent of men. The objectives of therapy are to minimize signs and symptoms as well as decrease the spread of infection to sexual partners. Mycoplasma genitalium in non-gonococcal urethritis has brought in much interest in the past years. Various research studies have exhibited that M. genitalium is a common cause for non-gonococcal urethritis where its eradication is synonymously related to improvement. Although research studies have revealed that Mycoplasma triggers symptomatic infections more than Chlamydia, it continues to be debatable whether mycoplasma urethritis can cause morbidity in men and women. DNA-based examinations with a $97 \%$ sensitivity have been established however, they are not yet commonly offered or used. M. genitalium is a meticulous microorganism that is debilitating to society. Numerous various other microorganisms have been linked in non-gonococcal urethritis. Like Mycoplasma, Urea-plasma types prevail in males with urethral signs and symptoms, however, their precise function as a pathogen has not been specified. Trichomonas varieties might likewise create urethral signs in males yet are challenging to identify. Herpes simplex virus (HSV) ought to be taken into consideration, specifically in patients with reoccurring signs or swelling of the meatus. Adenovirus has likewise been identified as a microorganism causing urethritis. Urethritis brought on by HSV or adenovirus is related to insertive sex amongst men who have sex with men [3].

\section{Epidemiology:}

Urethritis has an incidence of 4 million Americans yearly. The incidence of Neisseria gonorrhoea is approximated at over 600,000 cases each year, and the incidence of nongonococcal urethritis is about 3 million new cases each year. In one research of 424 men with symptoms and signs of severe urethritis, 127 (30\%) have infections of $\mathrm{N}$. gonorrhoea. In another study of 297 men with nongonococcal urethritis, 143 (48.1\%) had C. trachomatis as the infectious agent to cause urethritis. In 154 men presenting with non-chlamydial non-gonococcal urethritis, the organisms identified were: M. genitalium (22.7\%), M. hominis (5.8\%), U. urealyticum (19.5\%), U. parvum (9.1\%), H. influenzae $(14.3 \%)$, human adenovirus $(16.2 \%)$, N. meningitidis (3.9\%), T. vaginalis (1.3\%) and numerous kinds of herpes simplex virus $1(7.1 \%)$ as well as $2(2.6 \%)$. Urethritis is more common in men. Young age, unsafe intercourse, and having many sexual partners are all risk factors for urethritis. Neisseria gonorrhoea is among one of the most common venereal diseases and is the microbial cause for gonococcal urethritis in men and cervicitis in ladies. Chlamydia trachomatis is amongst one of the most typical venereal diseases. It is one of the most typical causes of nongonococcal urethritis in men and cervicitis in ladies. Mycoplasma genitalium is the causative organism in $15-20 \%$ of nongonococcal urethritis in men. Trichomonas vaginalis is a usual reason for nongonococcal 
urethritis in Africa. Regarding cases of NGU, Chlamydia trachomatis remains to be a key problem, although Trichomonas vaginalis and Mycoplasma genitalium are significantly identified as the causative pathogen. In addition, to the less frequent causes of urethritis like Urea-plasma parvum, Urea-plasma urealyticum, Mycoplasma hominis and Gardnerella vaginalis [4].

\section{History and physical:}

Urethritis is frequently asymptomatic; if symptomatic, however, the patient will present symptoms based on the causative pathogen. Signs and symptoms of urethritis might consist of dysuria, pruritus, burning sensation as well as discharge at the urethral meatus. Purulent discharge is typical of gonorrhoea. Dysuria alone prevails amongst chlamydia. If the patient has dysuria with painful genital abscess, then it is probably herpes simplex infection. Neisseria gonorrhoea infection typically presents as a large purulent or mucopurulent urethral discharge in males but can also be asymptomatic. In females, urethritis is frequently presented as cervicitis but can be asymptomatic as well. Dysuria is one of the most common signs seen in patients. Signs and symptoms in males can consist of frequency and urgency. Chlamydia trachomatis is most typically asymptomatic. Symptomatic individuals will present with dysuria and copious urethral discharge. A handful of individuals with chlamydial urethritis might develop reactive urethritis triad. Mycoplasma genitalium infections are typically asymptomatic. However, if symptomatic, signs might consist of dysuria, purulent or mucopurulent urethral discharge, urethral pruritus, balanitis, and posthitis. The urethral discharge is generally caused by this microorganism but it is not as evident as the Neisseria gonorrhoea infections. It can trigger severe recurrent urethritis in males. Herpes simplex infection normally causes extreme dysuria, and on physical exam, a restricted quantity of discharge can be found in addition to urethral meatitis and balanitis. Most of the people might not have herpetic sores on health examination. Adenovirus frequently causes extreme dysuria as opposed to urethral irritability caused by other causative organisms of nongonococcal urethritis. Adenovirus is normally transferred by oral sex presenting with upper respiratory tract infection symptoms during the winter season. Individuals normally do not report urethral discharge. Check-up generally reveals little urethral serous discharge; meatitis and balanitis are typically present and could show signs and symptoms of conjunctivitis. Recent studies suggest that enteric microorganisms are the causes of urethritis from anal direct exposure of gram-negative rods from urinary system infections or insertive anal sex. Hemophilus varieties, Neisseria meningitides, Moraxella catarrhalis, and also Streptococcus pneumonia are associated with insertive oral sex as causes for NGU [4].

\section{Clinical picture:}

Urethral discharge is the most suggestive sign of urethritis, existing in $71 \%$ of males with urethritis. Dysuria is the second most common sign, was reported in 59\% of men with urethritis. Discharge, as well as dysuria, are associated with the medical diagnosis of urethritis. Urethritis patients hardly ever report other signs ( $\leq 6 \%$ of males). As a matter of fact, just $4 \%$ of urethritis patients reported frequency, genital inflammation, itching, sores or smell, which were either not linked to urethritis or were part of the symptoms of another diagnosis not related to urethritis. This implies that signs and symptoms other than discharge or dysuria, point out to an alternate medical diagnosis (e.g. lesions caused by HPV and so on). Discharge, as well as dysuria, are one of the most vital signs to ask men being reviewed for urethritis. Signs of irritability, itching and lesions seem valuable when contributed to discharge and dysuria because these signs separately were not associated with urethritis. Inquiring about urinary frequency or genital odor, along with the previous signs and symptoms, identified just two additional cases of urethritis recommending there is little practicality in including them in the medical diagnosis. Genital inflam- mation, genital itchiness, genital sores, urinary system frequency, and urinary system odor were reasonably seldom, cumulatively making up just $13 \%$ of urethritis diagnoses. This suggests that males might have trouble verbalizing their signs precisely perhaps affected by their prejudgments of what signs and symptoms an STD "need to" seem like. As a streamlining method, it is recommended to use a wider, more incorporating term such as "genital discomfort" rather than "genital irritation" which would certainly determine $95 \%$ of urethritis diagnoses, when urethral discharge and dysuria are identified [5].

\section{Chlamydia trachomatis:}

Chlamydia is a distinct, tetracycline-sensitive, microbial pathogen. The failure of these microorganisms to manufacture ATP requires intracellular power parasitism for growth and duplication. 40 - 50 percent of NGU is caused by $\mathrm{C}$. trachomatis. The medical diagnosis of $\mathrm{C}$. trachomatis urethritis necessities cell culturing of the isolated microorganism or discovery of IgM-specific antibody by micro-immunofluorescence. Since neither of these methods is extensively offered, a presumptive medical diagnosis is made by excluding GU with urethral culture and Gram stain. Contrasted to GU, C. trachomatis urethritis is defined by a much longer incubation period, gradual onset 
of signs and symptom, clear or no discharge in more than 90 percent of cases and related to a higher socioeconomic status. Difficulties of C. trachomatis urethritis in males consist of Reiter's disorder in 1 - 2 percent, epididymitis in 3 percent, and involvement of prostate in 20 - 30 percent (mostly asymptomatic). On top of that, urethral stricture might arise from persistent C. trachomatis infection [6]

Significant effects of C. trachomatis infection in women and neonates have been recognized. Chlamydia trachomatis can be found in the endocervix of women with male sexual partners with C. trachomatis NGU. Although infection is mostly asymptomatic, purulent cervicitis, cervical dysplasia, pelvic inflammatory illness (PID), perihepatitis and acute urethral syndrome might arise. Five percent of women have shown to harbor $\mathrm{C}$. trachomatis and recent studies show culture and serology data that present it as the root cause of 20 - 35 percent of PID in the U.S. Vertical transmission of C. trachomatis was first identified in 1910 by Linder, that showed similar intracytoplasmic adhesion in mother's cervical scrapings and conjunctival scrapings from neonates with conjunctivitis. Evaluating information presently suggests that 10 percent of expectant females have cervical follicular conjunctivitis, nasopharyngitis, serous otitis media, and baby pneumonia. Prospective monitoring has shown that 35 - 50 percent of exposed babies develop conjunctivitis and 10 - 20 percent will develop pneumonia. Vertically transferred C. trachomatis is currently recognized as one of the most common sources of pneumonia in the first 6 months of life. Moreover, a ten-fold greater incidence of perinatal fatality has been reported in the mothers with $\mathrm{C}$. trachomatis infection compared with uninfected controls. The morbidity and mortality rate from C. trachomatis infection, require an aggressive method of treatment and control of NGU [6].

\section{Screening:}

The U.S. Preventive Services Task Force (USPSTF) has evaluated regular testing for chlamydia and gonorrhoea in males. Since difficulties in chlamydial infections in males are typically unusual, and evidence has showed that evaluating males doesn't cause a decline in negative outcomes in females, the USPSTF established in 2007 that there isn't enough evidence to suggest regular testing for chlamydia in men. Furthermore, asymptomatic gonorrhoeal infections are not commonly seen in men. In addition, gonorrhoeal infections have a low prevalence rate in the general populace which led the USPSTF's 2005 to advise against routine testing for gonorrhoeal infections in males especially in men with low risk of infection. However, the CDC advises numerous annual testing for high-risk groups like men who have sex with men. These include urethral/urine DNA screening for gonorrhoea and chlamydia in men who have had insertive sexual intercourse during the preceding year; testing for anal gonorrhoea and chlamydia in men who have had receptive anal intercourse during the previous year; as well as DNA swab for pharyngeal gonorrhoea in men who have had receptive oral sex during the previous year. Examining for pharyngeal chlamydial infection is not advised [1].

\section{Treatment:}

One of the most crucial steps in the proper management of urethritis is to identify whether N. gonorrhoea exists during the initial visit as this will help shape the treatment regimen. Despite some worries regarding the level of sensitivity, urethral smears for recognition of $\mathrm{N}$. gonorrhoea are advised for simultaneously recording inflammation and documenting the existence of intracellular diplococci. Patients without indicators of N. gonorrhoea infection are treated symptomatically. In males with severe signs and symptoms, the therapy must be started as quickly as the medical diagnosis is made, without waiting on the laboratory test results. In males with mild signs, a possible alternative is to evaluate the person after 3 - 7 days, when the outcomes of the NAAT(s) become available, as urethritis can be self-resolving without therapy. If lab examinations show positive results, or if its persistent urethritis, an ideal antimicrobial therapy targeting specific bacterium is advised and can be continued at the next visit. If the laboratory results come negative, only symptomatic patients that have evidence of urethritis should be treated. Any visible purulent discharge in symptomatic patients should warrant treatment with the proper antibiotics as well [7].

Preferably, therapy needs to be easy to take and effective with a microbiological cure $95 \%$ with a minimal side effect profile that will not cause disturbance of the patient's lifestyle. Nevertheless, analysing therapy efficiency is not simple. Observable inflammation might linger for a long time without persistent infection, and persistent infection might not lead to persistent NGU. Two current randomized trials from the United States observed that doxycycline $100 \mathrm{mg}$ x 2 for one week, and azithromycin 1 gram, had the same results in regards to their efficiency and clinical response rate. Mycoplasma genitalium therapy failure was commonly reported. A meta-analysis of chlamydia therapy revealed a $3 \%$ increase in cure rate with doxycycline compared to single-dose azithromycin for chlamydia infection. However, a $7 \%$ increase in treatment rate for urethritis was reported with doxycycline in 
comparison with azithromycin. There are 2 treatment regimens for azithromycin therapy, a one-gram dosage, or a 5 day-regimen with $500 \mathrm{mg}$ on the first day followed with by $250 \mathrm{mg}$ for the remaining days. Single-dose azithromycin regimen is believed to cause macrolide resistance in M. genitalium more frequently than the 5-day regimen. A preliminary dose of one gram azithromycin followed by $250 \mathrm{mg}$ on days 2 - 5 has been recommended. Nevertheless, no randomized research studies are contrasting the various treatment regimens in men with severe NGU. So, a 5 day regimen with initial dose of 1 gram is based on clinical experience. Doxycycline cure rate in C. trachomatis proved to be better than azithromycin with $40 \%$ cure rate of $\mathrm{M}$. genitalium without generating macrolide resistance and is used as first-line medication. Azithromycin is extensively used as first-line therapy for NGU yet it appears to have higher M. genitalium antimicrobial resistance. Azithromycin, particularly the solitary 1 gram regimen, will generate macrolide resistance in some $\mathrm{M}$. genitalium cases, and will certainly treat the macrolide sensitive strains. Hence, without an evaluation of treatment for M. genitalium and the traditional moxifloxacin therapy, the macrolide-resistant organisms will thrive and spread. Therefore, the proportion of macrolide-resistant strains in the population will increase and cause obstruction for future therapies. The substantial use of azithromycin 1 gram for the treatment of NGU for M. genitalium might be the reason for the declining cure rates. In Greenland, with a populace of 55,000, an extremely high incidence of NGU was reported as well as substantial use of the single-dose azithromycin. The macrolide resistance rate of $\mathrm{M}$. genitalium has been revealed to be $100 \%$, with a similar incidence of chlamydia and M. genitalium. Lymecycline or tetracycline hydrochloride do not cause photosensitivity and can be suggested if sunlight direct exposure cannot be avoided [8].

\section{Therapy of persistent NGU:}

If reinfection is not likely at follow-up, the patient has finished the first course of treatment, is symptomatic and has a urethral dis- charge, re-treatment needs to be offered. Any kind of therapy of persistent NGU needs to cover M. genitalium and $\mathrm{T}$. vaginalis and/or microbial vaginoses. Nevertheless, the following recommendations summarize the treatment based on guidelines and clinical experience. If doxycycline was used as first-line treatment, Azithromycin $500 \mathrm{mg}$ must be taken on the first day. After that $250 \mathrm{mg}$ of Azithromycin for 4 days plus metronidazole $500 \mathrm{mg}$ twice a day for 5 days. If azithromycin was used as first-line treatment, then doxycycline 100 mg must be taken twice a day for 7 days plus metronidazole $500 \mathrm{mg}$ two times daily for 5 - 7 days. Furthermore, for M. genitalium treatment, after azithromycin treatment failure, or in case of the macrolide-resistant pathogen, Moxifloxacin $400 \mathrm{mg}$ by mouth daily for 7 - 14 days must be prescribed. Moxifloxacin must be used with care and preserved for treatment failure which are believed to be macrolide-resistant M. genitalium, due to uncommon but serious negative hepatic reactions. In patients having acquired M. genitalium infection in South-East Asia, dual resistance to both macrolides and quinolones is present at around 10\%. Such infections are difficult to treat and only pristinamycin which is registered in France has proven to be reliable [8].

\section{Conclusion:-}

Urethritis is a sexually transmitted illness usually identified by urethral discharge or various other signs and symptoms such as itching, prickling, as well as complications in regular urinary flow. Microbiological examinations are obligatory to achieve good outcomes from the therapy. Several antibiotic therapies are commonly needed as a result of the high risk of numerous pathogens causing the pathology. Similarly, sexual partners must be examined and treated correspondingly. Several risk factors such as immunodeficiency, numerous sex partners, homo- and bisexuality, as well as alcoholic abuse might be connected to the condition. In these instances, using prophylactics and practicing safe sex is highly suggested.

\section{Bibliography:-}

1. Mellors JW. "Diagnosis and treatment of urethritis in men". Yale Journal of Biology and Medicine 56.2 (1983): $109-119$.

2. Tai E., et al. "Self-reported syphilis and gonorrhoea testing among men who have sex with men: national HIV behavioural surveillance system, 2003-5". Sexually Transmitted Infections 84.6 (2008): 478-482.

3. Brill JR. "Diagnosis and treatment of urethritis in men". American Family Physician 81.7 (2010): 873-878.

4. Young A and Wray AA. "Urethritis". StatPearls. Treasure Island (FL) (2019).

5. Jordan SJ., et al. "Defining the Urethritis Syndrome in Men Using Patient Reported Symptoms". Sexually Transmitted Diseases 45.7 (2018): e40-e42.

6. Edberg A., et al. "A comparative study of three different PCR assays for detection of Mycoplasma genitalium in urogenital specimens from men and women”. Journal of Medical Microbiology 57.3 (2008): 304-309.

7. Brihmer C., et al. "Efficacy and safety of azithromycin versus lymecyline in the treatment of genital chlamydial infections in women”. Scandinavian Journal of Infectious Diseases 28.5 (1996): 451-454.

8. Moi H., et al. "Management of non-gonococcal urethritis". BMC Infectious Diseases 15 (2015): 294. 